\title{
A System for Measuring Regional Surface Folding of the Neonatal Brain from MRI
}

\author{
Claudia Rodriguez-Carranza ${ }^{1}$, Pratik Mukherjee ${ }^{2}$, Daniel Vigneron ${ }^{2}$, \\ James Barkovich ${ }^{2}$, and Colin Studholme ${ }^{1,2}$ \\ ${ }^{1}$ NCIRE/VAMC, San Francisco, CA \\ ${ }^{2}$ Department of Radiology, University of California, San Francisco
}

\begin{abstract}
This paper describes a novel approach to in-vivo measurement of brain surface folding in clinically acquired neonatal MR image data, which allows evaluation of surface curvature within subregions of the cortex. This paper addresses two aspects of this problem. Firstly: normalization of folding measures to provide area-independent evaluation of surface folding over arbitrary subregions of the cortex. Secondly: automated parcellation of the cortex at a particular developmental stage, based on an approximate spatial normalization of previously developed anatomical boundaries. The method was applied to seven premature infants (age 28-37 weeks) from which gray matter and gray-white matter interface surfaces were extracted. Experimental results show that previous folding measures are sensitive to the size of the surface of analysis, and that the area independent measures proposed here provide significant improvements. Such a system provides a tool to allow the study of structural development in the neonatal brain within specific functional subregions, which may be critical in identifying later neurological impairment.
\end{abstract}

\section{Introduction}

The preterm birth rate, or percent of infants born at less than 37 completed weeks of gestation, has been on the rise in the last two decades in the USA. During 2003 the preterm delivery rate was $12.3 \%$ 6], which represents an increase of $16 \%$ since 1990 and more than $30 \%$ since 1981 . There is growing evidence that premature birth can result in structural and functional alterations of the brain, which are related to adverse neurodevelopmental outcome later in life [7,8. Some of the challenges that preterm infants face range from spastic motor deficits (cerebral palsy) [17, impaired academic achievement [5, 3], and behavioral disorders [9]. However, the etiology of cerebral abnormalities that underlie these common and serious developmental disabilities is not entirely understood [8]. The wider availability of clinical in vivo magnetic resonance imaging of neonatal brain anatomy, provided by systems which make use of an MRI compatible incubator, creates a new opportunity to quantify brain development.

In this work we are particularly interested in the study of cortical folding, or gyrification, in preterm infants. We would like to examine folding within specific subregions of the cortex that may correspond to the development of particular functional areas. In our earlier work we have applied previously proposed global 
measures of folding to surfaces extracted from neonatal MRI, and showed their use in tracking global age changes [13. The main limitation was that the previously proposed measures are heavily dependent on the area of the surface being examined. Hence those measures cannot be directly applied to subregions of the brain.

In this paper we describe an approach to creating area-independent global measures of folding and an approach to partitioning the early cortex into regions to which these area-independent folding measures can be applied. We show the influence of the normalization on synthetic data and then apply the measures to surfaces extracted from MR images of seven premature infants with ages from 28-37 weeks.

\section{Method}

Several global 3D brain surface folding measures have previously emerged. Some are based on surface principal curvatures $\left(k_{1}, k_{2}\right)$, mean curvature $\left(H=\frac{1}{2}\left(k_{1}+\right.\right.$ $\left.k_{2}\right)$ ), or Gaussian curvature $\left(K=k_{1} k_{2}\right)$. Examples are folding index $(F I)$ [4], the intrinsic curvature index $(I C I)$ 4], $L^{2}$ norm of the mean curvature $(M L N)$ ], $L^{2}$ norm of Gaussian curvature $(G L N)$ [1], and average curvature [11]; global shape index $(G S)$ and global curvedness $(G C)$ were defined in 13 based on the local shape descriptors curvedness (c) [10] and shape index (s) [10]. Other measures are non-curvature based such as the gyrification index [18, calculated as the ratio of the entire cortical contour of the brain to the superficially exposed contour, and roundness $(\mathrm{Rn})$, based on surface area $(A)$ and volume $(V)$ (it is also described as surface complexity [11] and isoperimetric ratio [1]). A list of current global 3D measures of gyrification is shown on the left column of Table 1 . These expressions are normalized to yield the unit value for a sphere 13 .

\subsection{Size-Independent Measures of Surface Folding}

Examination of the form of the current global measures in Table 1 reveals a critical dependency on surface area, with the exception of shape index and gyrification index. This can be better illustrated with the following example, using $M L N$ to measure folding. Take a whole sphere of radius Ro, half a sphere of radius $R o$, and a whole sphere of half that radius. The three objects have the same surface complexity, hence a measure of global folding should yield identical results. By definition, $H=1 / R$ at each point in a sphere. Surface folding yields $M L N=\frac{1}{4 \pi} \times 4 \pi R o^{2} \times(1 / R o)^{2}=1$ for the whole sphere with radius $R o$ and also for whole sphere with radius $R o / 2: M L N=\frac{1}{4 \pi} \times\left(4 \pi \frac{R o}{2}\right)^{2} \times(2 / R o)^{2}=1$. On the other hand, $M L N$ for the half sphere of radius $R o$ is half that value: $M L N=\frac{1}{4 \pi} \times \frac{1}{2} 4 \pi R o^{2} \times(1 / R o)^{2}=\frac{1}{2}$. This demonstrates the dependency of the measure on the size of the surface of analysis. Normalizing $M L N$ with the surface area does not alleviate the problem: $M L N=\frac{1}{A} \sum_{A} H^{2}=\frac{1}{4 \pi R o^{2}}\left(4 \pi R o^{2}\right)(1 / R o)^{2}$ $=\frac{1}{R o^{2}}$ (whole sphere of radius $R o$ ). We propose two normalization factors: 1) $T=3 \mathrm{~V} / \mathrm{A}$ and 2) $\overline{|H|}=\frac{1}{A}\left(\left|H^{+}\right|+\left|H^{-}\right|\right)$. The resulting $T$-normalized and $H$-normalized expressions ( $T$-measures and $H$-measures, respectively) are shown 
Table 1. Measures of surface folding

\begin{tabular}{|c|c|}
\hline $\begin{array}{c}\text { Current measures } \\
\text { Area-dependent } \\
M L N=\frac{1}{4 \pi} \sum_{A} H^{2}[1] \\
G L N=\frac{1}{4 \pi} \sqrt{A * \sum_{A} K^{2}}[1] \\
I C I=\frac{1}{4 \pi} \sum_{A} K^{+}[4] \\
F I=\frac{1}{4 \pi} \sum_{A} a k[4] \\
G C=\frac{1}{\sqrt{A 4 \pi}} \sum_{A} c[13 \\
\bar{U}=\frac{1}{A} \sum_{A} U[1] \\
R n=\frac{A}{\sqrt[3]{36 \pi V^{2}}}[1] \\
\text { Area-independent } \\
G S=\frac{1}{A} \sum_{A} s[13 \\
\text { Gyrification Index }[18\end{array}$ & $\begin{aligned} & \text { New measures } \text { Area-independent } \\
& M L N_{T}=\frac{T^{2}}{A} \sum_{A} H^{2} \\
& G L N_{T}=T \sqrt[4]{\frac{1}{2} \sum_{A} K^{2}} \\
& I C I_{T}=T \sqrt{\frac{1}{A} \sum_{A} K^{+}} \\
& F I_{T}=T \sqrt{\frac{1}{A} \sum_{A} a k} \\
& G C_{T}=T \sqrt{\frac{1}{A} \sum_{A} c} \\
& \overline{U_{T}}=\frac{T}{A_{U}} \sum_{A} U \\
& S H 2 S H=T \sum_{A} H^{2} / \sum_{A} H \\
& S K 2 S K=T \sqrt{\sum_{A} K^{2} / \sum_{A} K} \\
& M L N_{H}=\frac{1}{\mid \overline{|H|}} \sqrt{\sum_{A} H^{2} / A} \\
& G C_{H}=\frac{1}{A \overline{|H|}} \sum_{A} c \\
& A F_{U+}=A_{U+} / A \\
&\end{aligned}$ \\
\hline $\begin{array}{l}\text { Notation: } c=\sqrt{\frac{1}{2}\left(k_{1}^{2}\right.} \\
\frac{2}{\pi} \arctan \frac{k_{2}+k_{1}}{k_{2}-k_{1}} \text { (shape in } \\
T=3 \frac{V}{A}, \text { and } \overline{|H|}=\frac{1}{A}(\mid H \\
\text { identified with }{ }^{+} \text {or }^{-}, U\end{array}$ & $\begin{array}{l}(\text { curvedness }[10]), \quad s= \\
10]), a k=\left|k_{1}\right|\left(\left|k_{1}\right|-\left|k_{2}\right|\right) \text {, } \\
\left.\left|H^{-}\right|\right) \text {. A curvature's sign is }\end{array}$ \\
\hline
\end{tabular}

in the right column of Table1, For a sphere, $T=R o, \overline{|H|}=1 / R o$, and it can be verified that $M L N_{T}=1$ for the three sphere cases. Similarly for $G L N_{T}, I C I_{T}$, $F I_{T}, G C_{T}, \overline{U_{T}}, M L N_{H}$, and $G C_{H}$.

In addition, we defined three new area-independent folding measures: $S H 2 S H, S K 2 S K 2$, and $A F_{U}$. The first two were based on the rationale that measures composed of ratios of local curvature factors would intrinsically eliminate the dependence on area. The latter was based on the idea that a reasonable characterization of the degree of folding in a brain surface is the fraction of the surface which contains convex folds (gyri). This can be mathematically characterized by looking at the relative portion of the surface which has positive mean or Gaussian curvature. For an undeveloped brain, the fraction would be large, and it would progressively decrease as the concave folds (sulci) appear. The definition of the three expressions is shown in Table 1.

\subsection{Cortical Partitioning Using Spatial Normalization}

In order to study changes in regional surface folding across different subjects, we need to be able to identify boundaries from the structural image data which consistently partition the brain surface in different subjects at different stages of development. In this initial work we have used a single reference anatomy which 
was partitioned into frontal and non-frontal regions based on the presence of the post-central gyrus. The sulci around this region develop earlier in the brain folding process and provide a landmark to allow us to study regional folding over a range of ages after this is present. The approximate partitioning into left and right frontal cortex and non-frontal cortex was marked on a single reference MRI. A multi-resolution B-Spline based spatial normalization, adapted from that described in 15, 16 and as used in 2, was then applied to estimate a spatial transformation mapping from this reference to each subject MRI being studied. This transformation was then numerically inverted to allow the assignment of the nearest reference label to a given subject image voxel, which brought the voxelwize partitioning of the reference anatomy to the same space as the surface extracted from each individual. The partitioning was then used to constrain the voxelwize evaluation of the folding measures.

\subsection{Data and Image Processing}

Synthetic Image Data. The measures were tested on synthetic binary shapes constructed from the following expression: $x=\rho \sin (\phi) \cos (\theta), y=\rho \sin (\phi) \cos (\theta)$, $z=\rho \cos (\phi), \rho=k *\left(\sin ^{b}(a \phi)+\cos ^{d}(c \phi)+\sin ^{f}(e \theta)+\cos ^{h}(g * \theta)\right)(k$ is a scaling factor). Coordinates $x, y, z$ are computed in the range $\phi \in[0, \pi]$ and $\theta \in[0,2 \pi]$ given a set of user-defined parameters $a, \ldots, h$. The voxels within the shape were assigned a value of 1000 and background voxels a value of 0 .

Human Neonatal Images. High resolution $(0.703 \times 0.703 \times[1.5-2.0] \mathrm{mm})$ 3D T1 weighted SPGR images were acquired on seven premature infants using a 1.5T GE MRI scanner with an MRI compatible incubator. The gestational ages of the premature infants ranged between 24-31 weeks. The postmenstrual ages (gestational age + postnatal age) at the time of acquisition were 28-37 weeks. A subsequent scan for three of the infants was available, hence a total of ten brains was processed. The outer gray matter and the gray-white matter interface 1 surfaces were manually extracted as described in 13 using the rview software package [14]. A value of 1000 was assigned to each segmented brain voxel and 0 to the background.

Computation of Surface Folding. Each binary volume (the segmented neonatal brain or the synthetic shape) was supersampled using voxel replication to prevent loss of fine scale features $(2 \times 2 \times 4$ for neonatal images and $2 \times 2 \times 2$ for synthetic images). A voxelwize approach to surface curvature estimation from iso-surfaces, derived from that developed by Rieger [12, was then employed. It avoids the need of a parametric model. A summary of the sequence of steps (described in detail in [13]) is as follows: 1) computation of the image gradient $g=\nabla f(x, y, z)$ of the replicated binary volume; 2$)$ computation of the gradient structure tensor (GST) [12] defined as $T=\overline{g g^{t}} ; 3$ ) calculation of the eigenvectors $v_{1}, v_{2}, v_{3}$ of $T$ and the mapping $\left.M\left(v_{1}\right)=\frac{v_{1} v_{1}^{t}}{\left\|v_{1}\right\|} ; 4\right)$ computation of the principal

\footnotetext{
${ }^{1}$ The terms gray-white matter interface and white matter will be used interchangeably through the text.
} 
curvatures from $\left|k_{1,2}\right|=\frac{1}{\sqrt{2}}\left\|\nabla_{v_{2,3}} M\left(v_{1}\right)\right\|_{F}$ (Fröbenius norm); the sign of $k_{1}$ and $k_{2}$ is determined from the Hessian matrix of the image and the eigenvectors $v_{2}$ and $v_{3}$. The curvatures $k_{1}$ and $k_{2}$ were then used to compute mean $(H)$ and Gaussian $(K)$ curvature and the folding measures of Table 1 Each step involves image smoothing or differentiation. For all images, the $3 \mathrm{D}$ filters used at the $i$ th step had a full-width at half maximum $\left(f_{i}\right)$ of: $f_{1}=2.1 \mathrm{~mm}$ (for synthetic images, $\left.f_{1}=3 \mathrm{~mm}\right), f_{2}=1 \mathrm{~mm}, f_{3}=2 \mathrm{~mm}, f_{4}=1 \mathrm{~mm}$. The convolution of the first step creates a volume with voxel values between 0 and 1000, which represents percentage occupancy. The surface of interest, i.e. the one on which the global measures of folding were calculated, was taken to be the iso-surface with at least $50 \%$ occupancy and satisfying 6 -connectivity with the background. The rationale behind the choice of values was to create a smooth surface that would yield smooth variations in curvature.

\section{Results}

Firstly we applied the measures to subregions of synthetic surfaces. Figures 1 (a), (b), (c), and (d) show the map of (local) mean curvature on four subregions of the shape with parameters $\mathrm{a}=1, \mathrm{~b}=4, \mathrm{c}=1, \mathrm{~d}=3$, $\mathrm{e}=1, \mathrm{f}=2, \mathrm{~g}=1, \mathrm{~h}=4$, and scale factor $k=10$. The numerical results that follow are shown in the order of appearance in Figure 1. The area of each subregion was: 3626.5, 1814.5, 789, $772.9 \mathrm{~mm}^{2}$. MLN yielded 2.32, 1.17, 0.72, 0.43 ; note that $M L N$ for the whole shape is about twice the value of $M L N$ for the half shape. This exemplifies the dependency of the old measures on the size of the region of analysis. On the other hand, the new $M L N_{T}$ for the whole and half spheres was the same: $1.37,1.37,1.08,0.91$. This is reasonable since the partition was at an axis of symmetry.

We then applied the new measures globally to surfaces from the ten MRI studies to investigate their response to folding with age using linear regression of their values against age. Figure 1(e) shows the map of (local) mean curvature on white matter. Figure 2 shows the graphs of five of the global folding measures against postmenstrual age, for both cortical gray matter and white matter (due to space limitations, only five graphs are shown). The measures $G S$ and $A F_{H^{+}}$ decrease with age, as opposed to the other measures. This is because the younger the infant, the less folded is the brain and the shape is closest to that of a sphere ( $G S=1$ and $A F_{U}=1$ for a sphere). The measures with greater goodnessof-fit for gray matter were T-normalized measures: $F I_{T}, \overline{H^{+}}$, and $\overline{K^{-}}\left(R^{2}=\right.$ $[0.86,0.89])$; for white matter, it was $G S$ (global shape index), $A F_{H^{+}}, G C_{H}$, and $M L N_{H}\left(R^{2}=[0.95,0.96]\right)$. The goodness-of-fit for gray matter and white matter (in that order) for all measures was: $M L N_{T}(0.80,0.04), G L N_{T}(0.79$, $0.14), I C I_{T}(0.83,0.0), F I_{T}(\mathbf{0 . 8 9}, 0.13), G C_{T}(0.85,0.01), \overline{H^{-}}(0.70,0.06)$, $\overline{H^{+}}(\mathbf{0 . 8 6}, 0.13), \overline{K^{-}}(\mathbf{0 . 8 8}, 0.25), \overline{K^{+}}(0.83,0.0), G S(0.69, \mathbf{0 . 9 6}), \mathrm{SH} 2 \mathrm{SH}(0.77$, $0.17)$, SK2SK $(0.66,0.37), M L N_{H}(0.48, \mathbf{0 . 9 5}), G C_{H}(0.55, \mathbf{0 . 9 5}), A F_{H^{+}}=(0.69$, 0.95), $A F_{K^{+}}=(0.51,0.0)$.

Finally, we applied the measures to examine their relationship with age regionally on gray matter, over frontal and non frontal cortical regions (subdivided 


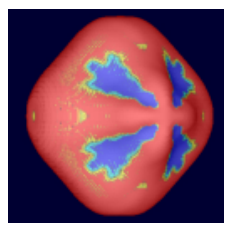

(a)

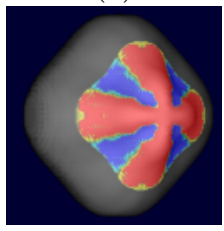

(c)

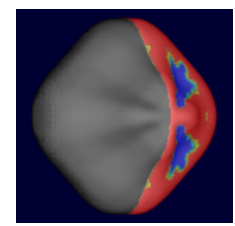

(b)

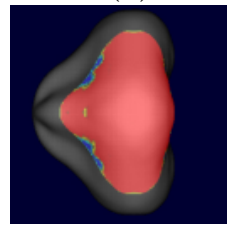

(d)

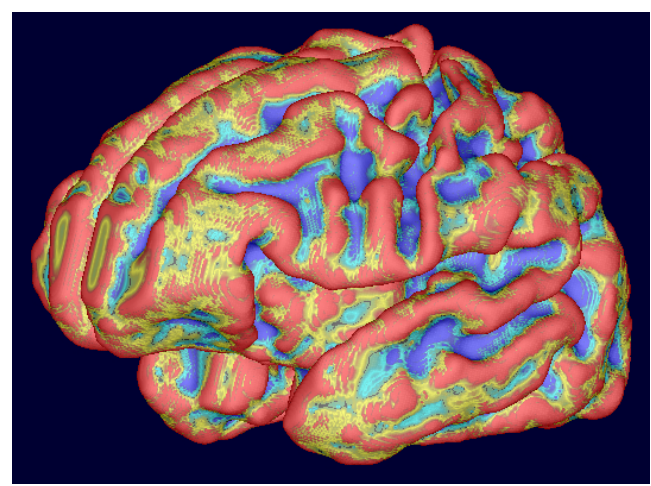

(e)

Fig. 1. Map of mean curvature $(H)$ for the synthetic shape: (a) whole surface, (b) half the surface, (c) subregion I, (d) subregion II and (e) for white matter of a 32.4 week old infant. $H$ varies within $[-0.15,0.15]$.
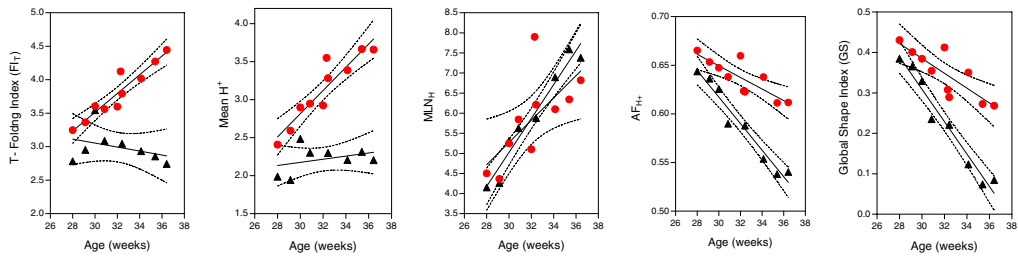

Fig. 2. Plots of five folding measures on cortical gray matter ( $\bigcirc$, ten studies) and white matter $(\triangle, 8$ studies) against age. Linear fits and $95 \%$ confidence intervals are shown.

into left and right). Plots for area and four folding measures are shown in Figure 3 . The rate of change of $F I_{T}, \overline{H^{+}}$, and $I C I_{T}$ is positive with age and, hence, with cortical folding. The results show that frontal regions had a smaller area but larger degree of folding than non frontal regions. Except for $F I_{T}$ on the left frontal subregion of the cortex, linear fits on all subregions had similar $R^{2}$ to the linear fits for the whole brain. The graph of GLN exemplifies the dependency of the previously defined measures of the area of analysis.

\section{Discussion}

An understanding of the cortical folding process in the development of premature infants may be important in explaining and predicting abnormal neurological outcome. The use of formal mathematical descriptions provides a more quantitative tool to study the folding process than is available with simple visual evaluation of MRI scans. The long term goal of our research is to create a model to track preterm in vivo neonatal brain cortical development that will help characterize normal gyrification and departures from it. 

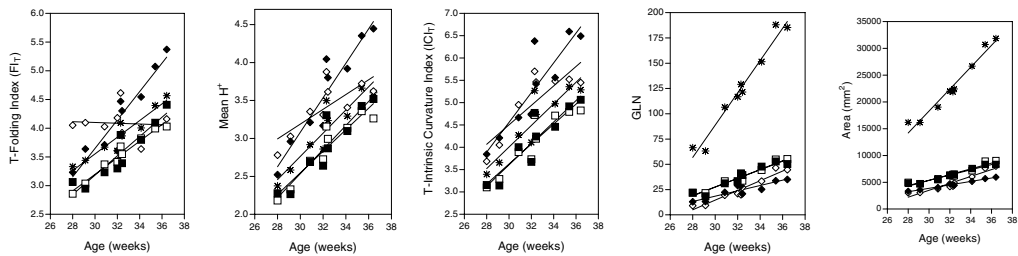

Fig. 3. Plots of four global folding measures and area against age for the frontal $(\diamond)$ and non-frontal $(\square)$ regions of gray matter. Results for the whole surface (*) are included. Filled shapes represent the left side of the brain, empty ones the right side. Linear fits are shown.

In this work we have shown that most previously proposed folding measures are dependent on the size of the surface patch on which they are applied. To alleviate this problem we examined two approaches to normalization of the measures and proposed new forms of measure, which satisfy the requirements of area-independence. These were evaluated on synthetic image data and neonatal brain surfaces. We evaluated the ability of the measures to detect change in normal aging by calculating the linear regression of each measure with age globally and locally. Globally, gray-white matter interface folding tracked best with age. No single measure rated consistently well for both surfaces: $F I_{T}, \overline{H^{+}}, \overline{K^{-}}$had the best fit for cortical gray matter, while $M L N_{H}, G C_{H}, G S$, and $A F_{H^{+}}$did for the gray-white matter interface. Locally, the measures were able to capture folding differences between frontal and non frontal regions. The proposed normalization factors $T$ and $\overline{|H|}$ opened the possibility to adapt previously defined measures to quantify gyrification in subregions of the brain.

We need to further investigate the sensitivity and validity of the measures in a larger cohort, containing both normal and pathological cases. In conclusion, the proposed new normalized measures allow area-independent assessment of folding which provides the ability to study regional gyrification. Such measures will provide a new tool in assessing global and local surface folding which is independent of the overall surface area, and are hence applicable to developing a model that tracks development in premature infants.

\section{Acknowledgments}

This work was funded by the NIH grant R01 MH65392.

\section{References}

1. P. G. Batchelor, A. D. Castellano Smith, D. L. G. Hill, D. J. Hawkes, T. C. S. Cox, and A. F. Dean. Measures of folding applied to the development of the human fetal brain. IEEE Transactions on Medical Imaging, 21(8):953-965, August 2002.

2. V. Cardenas, L.L. Chao, R. Blumenfeld, and et al. Using automated morphometry to detect association between ERP latency and structural brain MRI in normal adults. Human Brain Mapping, 25(3):317-327, 2005. 
3. R. W. I. Cooke, L. Foulder-Hughes, D. Newsham, and D. Clarke. Ophtalmic impairment at 7 years of age in children born very preterm. Archives of Disease in Childhood Fetal and Neonatal Edition, 89:249-253, 2004.

4. D.C. Van Essen and H. A. Drury. Structural and functional analyses of human cerebral cortex using a surface-based atlas. The Journal of Neuroscience, 17(18):7079$7102,1997$.

5. M. Hack, D. J. Flannery, and et al. Outcomes in young adulthood for very-lowbirth weight infants. New England Journal of Medicine, 346(3):149-157, 2002.

6. B. E. Hamilton, J. A. Martin, and P. D. Sutton. Births: Preliminary data for 2003. National Vital Statistics Reports, 53(9), 2004.

7. P. S. Hüppi and et al. Structural and neurobehavioral delay in postnatal brain development of preterm infants. Pediatric Research, 39(5):895-901, 1996.

8. T. E. Inder and et al. Abnormal cerebral structure is present at term in premature infants. Pediatrics, 115(2):286-294, 2005.

9. T. E. Inder, S. J. Wells, N. B. Mogridge, C. Spencer, and J. J. Volpe. Defining the nature of the cerebral abnormalities in the premature infant: a qualitative magnetic resonance imaging study. Journal of Pediatry, 143:171-179, 2003.

10. J. J. Koenderink and A. J. van Doorn. Surface shape and curvature scales. Image and Vision Computing, 10(8):557-564, 1992.

11. V. A. Magnotta, N. C. Andreasen, and S. K. Schultz et al. Quantitative in vivo measurement of gyrification in the human brain: changes associated with aging. Cerebral Cortex, 9:151-160, 1999.

12. B. Rieger, F. J. Timmermans, L. J. van Vilet, and P. W. Verbeek. On curvature estimation of iso surfaces in $3 \mathrm{D}$ gray-value images and the computation of shape descriptors. IEEE Transactions on Pattern Analysis and Machine Intelligence, 26(8):1088-1094, August 2004.

13. C. Rodriguez-Carranza, F. Rousseau, B. Iordanova, O. Glenn, D. Vigneron, J. Barkovich, and C. Studholme. An iso-surface folding analysis method applied to premature neonatal brain development. In Proc. SPIE Medical Imaging: Image Processing, volume 6144, pages 529-539, 2006.

14. C. Studholme. http://rview.colin-studholme.net/.

15. C. Studholme, V. Cardenas, and et al. Accurate template-based correction of brain MRI intensity distortion with application to dementia and aging. IEEE Transactions on Medical Imaging, 23(1):99-110, 2004.

16. C. Studholme, V. Cardenas, and et al. Deformation tensor morphometry of semantic dementia with quantitative validation. Neuroimage, 21(4):1387-1398, 2004.

17. J. J. Volpe. Neurologic outcome of prematurity. Archives of Neurology, 55:297-300, 1998.

18. K. Zilles, G. Schlaug, M. Matelli, G. Luppino, and et al. The human pattern of gyrification in the cerebral cortex. Anatomy and Embriology, 179:173-179, 1988. 